\title{
Publisher's Note: Test on the effectiveness of the sum over paths approach in favoring the construction of an integrated knowledge of quantum physics in high school [Phys. Rev. Phys. Educ. Res. 13, 010101 (2017)]
}

Massimiliano Malgieri, Pasquale Onorato, and Anna De Ambrosis (Received 19 January 2017; published 3 February 2017)

DOI: 10.1103/PhysRevPhysEducRes.13.019901

This paper was published online on 3 January 2017 with an incorrect volume number in the article identifier. The correct volume is "13." The paper has been corrected as of 19 January 2017. 